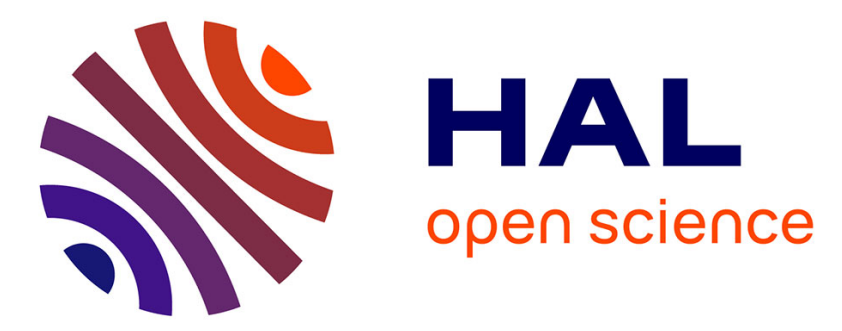

\title{
STRUCTURE OF THE GIANT ANGLE DIPOLE
}

\author{
R. Hilton, S. Iwasaki, H. Mang, P. Ring, M. Faber
}

\section{To cite this version:}

R. Hilton, S. Iwasaki, H. Mang, P. Ring, M. Faber. STRUCTURE OF THE GIANT ANGLE DIPOLE. Journal de Physique Colloques, 1987, 48 (C2), pp.C2-59-C2-63. 10.1051/jphyscol:1987209 . jpa00226474

\section{HAL Id: jpa-00226474 https://hal.science/jpa-00226474}

Submitted on 1 Jan 1987

HAL is a multi-disciplinary open access archive for the deposit and dissemination of scientific research documents, whether they are published or not. The documents may come from teaching and research institutions in France or abroad, or from public or private research centers.
L'archive ouverte pluridisciplinaire HAL, est destinée au dépôt et à la diffusion de documents scientifiques de niveau recherche, publiés ou non, émanant des établissements d'enseignement et de recherche français ou étrangers, des laboratoires publics ou privés. 
R.R. HILTON, S. IWASAKI, H.J. MANG, P. RING and M. FABER*,

Fhysics Department, Techntcal University Munich, D-8046 Garching. F.R.G.

"Institute of Nuclear Physics, Technical University, A-1020 wien, Austria

\section{ABSTRACT}

An extensive investigation of the angular analogue to the Giant Dipole, the "Giant Angle Dipole", undertaken within the framework of a self-consistent QRPA formalism employing Skyrme forces, has revealed a number of facets about this state. In the case of ${ }^{150} \mathrm{Gd}$, the QRPA solutions obtained showed fragmentation of the $B(M 1)$ strength occurs without the need to postulate triaxiallity or octupole deformations. Overlaps unambiguously reveal the dominant component of the fragmented state found at 3.41 Mev, having $B M(1)=1.49 \mu_{N^{2}}$ with little spin flip contribution, to be a contra angular rotational oscillation of the proton and neutron densities, together with isovector plus isoscaler admixture of two orthogonal shears, in agreement with the ascillator model. The implications of this state as a tool in nuclear structure physics are discussed.

\section{INTRODUCTION}

The identification of the long conjectured "Giant Angle Dipole" $/ 1-7 /$, in recent $e, e^{\circ}$ scattering experiments on ${ }^{15 \Delta} \mathrm{Gd} / 8 /$, and its confirmation in independent photo excitation and forward angle $P, p$. measurements 110-12/, have opened the way for a new tool in nuclear structure physics, with the promise of gaining hitherta inaccessible information. In the last three years well over 100 theoretical and experimental studies of this state have been undertaken, during the course of which many names have been given to this mode viz: positive parity quadrupale resonance; $K=1$ * resonance; mixed symmetry mode; scissor mode; giant magnetic dipole; giant $M 1$ mode and nuclear wobble. However here we shall refer to it by its original name, the "Giant Angle Dipole", for further references c.t. $14,5 /$. The picture of collective motion in which proton and neutron densities vibrate against one another in angular fashion about an axis perpendicular to the symmetry axis of the nucleus has, almost without exception, been the common basis of these many investigations. However, such a picture, built in strict analogy with the Giant Dipole, is quantum mechanically inconsistent with the description of a coherent low energy collective state /2-5/.

\section{THE OSCILLATOR MODEL}

For a large particle number fermion system contained in a deformed oscillator, collective coordinates $J$ and momenta $\mathrm{II}$ corresponding to the Giant Angle Dipole can be found. In terms of these variables, the shell model Hamiltonian may be shown to display the form $12,3 /$

$$
H \rightarrow H_{0}=\pi=/ 2 B+C J^{2} / 2+H_{A}\left(q_{1}, p_{1}\right)
$$


in which the variables $\left\{q_{1}, p_{1}\right\}$ describe remaining degrees of freedom of the system commuting with 3 and $\pi$. The ground state is then $\varphi_{0}(q) \chi_{0}(\xi)$ and wavefunctions of the form of $\varphi_{0}(q) \chi_{N}(3)$ may be interpreted as Giant Angle Dipole collective excitations, in much the same spirit as for the Giant Dipole/14/.

From the model we learn that this excitation is a positive parity quadrupole excitation carrying isospin $T=1$ (with small isospin Txo admixture), comprising a linear combination of angular momentum z-projection $M= \pm 1$. (For a self-conjugate nucleus it reverts to pure $T=1)$. The excitation energy of the Giant Angle Dipole turns out to be low, around 3-4 Mev for the well deformed rare earths. Transition strengths may also be assessed, and in the case of ${ }^{136} \mathrm{Gd}$ we gain a $B(M 1)$ value around $7.5 \mu^{2}$, with no spin flip contributions since we have a spin saturated system. The effects of correlations modify these values somewhat $/ 3 /$. In the case of ${ }^{30} \mathrm{Gd}$ an energy at 3 Mev and a E(M1) value of around $4.9 \mu^{2}$ are expected. The underlying physical nature of this state, digclosed by examining the Giant Angle Dipole generators, show it to be a $\Delta N=0$ excitation, comprising a sum of motions: a pure contra angular rotational oscillation together with an isovector plus isoscaler admixture of two orthogonal shears. The picture of pure contra rotation at the heart of many models, it becomes apparent, is incompatible with the description of a low energy coherent state, as the generators of such motion lead to collective excitations built out of linear combinations of single particle states caming from different oscillator shells, i.e. $\Delta N=0$ and $\Delta N=2$. The osciliator model reveals how to marry the picture of this low energy collective excitation with an underlying single particle structure consistent with quantum mechanics.

\section{MICRDSCOPIC DESCRIPTION}

A high resolution search of the (e,e') scattering spectrum of ${ }^{130} \mathrm{Gd}^{\prime}$ carried out at the Darmstadt LINAC revealed a sharp $1^{\circ}$ resonance at 3.07 Mev, carrying a $B(M 1)$ strength of $1.3 \mathrm{H}^{2} / 8,7 /$. The marked discrepancy between the observed $B(M 1)$ strength and our collective model value strongly implied a fragmented structure to the 1 , and a microscopic formalism was required in which the interplay between the single particle and collective aspects of the system could be treated. To this end, an extensive investigation within the framework of a self-consistent QRPA formalism employing Skyrme forces was undertaken. A fully self-consistent H.F. calculation generating the single particle energies and states served as the input for the QRPA formalism. Further details of notation and formalism are given in $14,5,15 /$.

For arbitrary variations $\delta Q$, we gain from the Schrödinger equation,

$$
\left\langle 0:\left[\delta Q,\left[H, Q^{*} .3\right]: 0\right\rangle=\left(E_{,},-E_{0}\right\rangle\left\langle 0:\left[\delta Q, Q^{*},\right]: 0\right\rangle\right.
$$

Restricting ourselves to collective operators $Q$ of the form

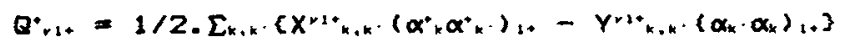

we gain the QRPA equations

$$
\left(\begin{array}{cc}
A & B \\
-B^{*} & -A^{*}
\end{array}\right)\left(\begin{array}{l}
X^{+1+} \\
Y^{*+1}
\end{array}\right)=w_{+1+}\left(\begin{array}{l}
X+1+ \\
Y^{+1+}
\end{array}\right)
$$

The eigenvalues $w_{1,}$ and eigenvectors corresponding to (4) were then calculated using ( 3 ). Shown in $f i g .1$ are the $B(M 1)$ strengths found for each state up to 5 Mev, calculated from the expression 


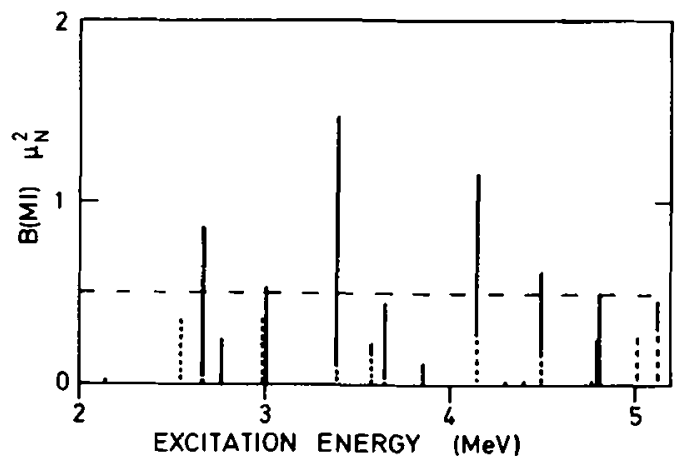

Fig. 1 B (M1) strength

Here |HFB> is the HFB ground state. In fig.l the spin flip contributions are shown as dotted lines. The height of typical two quasi-particle grass is indicated by the horizontal dashed line. We observe fragmentation of the $B(M 1)$ strength has occurred without the need to introduce either triaxial or octupole deformations. The dominant component in the spectrum is seen at $3.41 \mathrm{Mev}$, having a $B$ (M1) strength of $1.49 \mu_{N}^{2}$ with little spin flip admixture. The physical nature of this state was obtained from calculating the overlap,

$\left\langle H F B:\left[\left(J_{2}-J_{N}-\eta\left(S_{2}-S_{N}\right)\right\}, Q^{+}{ }_{N 1+}\right]: H F B\right\rangle *$

$\left\langle H F B ;\left[J_{1}-J_{N}-\eta\left(S_{2}-S_{N}\right)\right]^{2}[H F B\rangle-1 / 2\right.$

(6)

as a function of the shear admixture parameter $\eta$. As seen in fig.2 the overlap with a state describing pure contra rotation ( $\eta=0$ ) is already over 53\%. However, the maximum overlap accurs at a non vanishing $\eta$ value ( $\eta_{\max }=0.25$ ), unambiguously demonstrating that the isovector component of this state comprises a pure contra rotation plus a shear, as had already been anticipated from the oscillator model. In fact the microscopic description indicates more shear admixture than the oscillator value $\left(\eta_{0}\right)$.

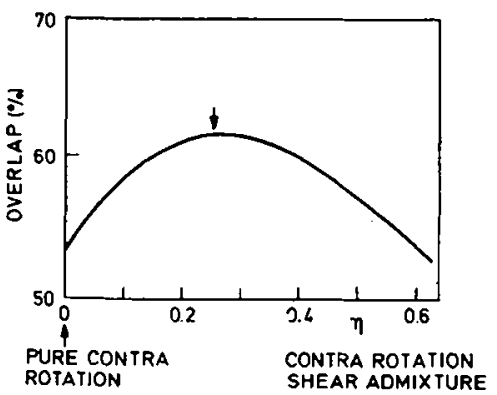

Fig. 2 Overlap shown as a function of $\eta$

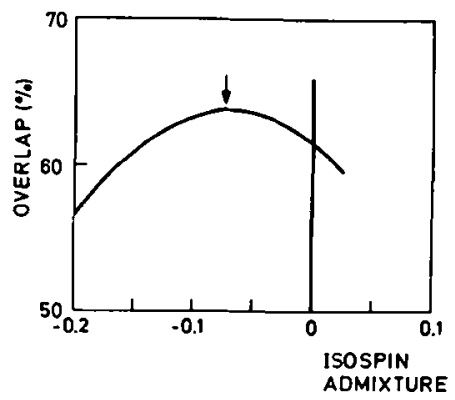

Fig. 3 Overlap shown as a function of $\varepsilon$

The investigation was extended and the overlap,

$\left\langle H F B:\left[\left\{J_{2}-J_{N}-\eta_{\max }\left(S_{2}-S_{N}\right)+\varepsilon\left(S_{2}+S_{N}\right)\right\}, Q^{*}, \ldots\right]\right.$ iHFB $\rangle *$

$\left\langle H F B !\left[J_{z}-J_{N}-\eta_{\max }\left(S_{2}-S_{N}\right)+\varepsilon\left(S_{2}+S_{N}\right)\right]^{2}: H F B>-1 / 2\right.$ 
calculated as a function of the isoscaler mixing parameter $\varepsilon$ (with $\eta$ $f i x e d$ at $\left.\eta_{\max }\right)$. As seen from $f i g .3$, the overlap reaches its maximum value for $\left(\varepsilon_{\text {max }}=-0.075\right.$ ), showing an isoscaler admixture which is also larger than that given by the oscillator $\left(\varepsilon_{0}=-0.033\right)$ at the same deformation.

Fig.4 illustrates the geometric picture of the motion which emerges for the dominant component. The isoscaler admixture reflects the fact that the protons and neutrons do not deform by the same amount during their motion. The excited state thus encapsulates the properties anticipated of the Giant Angle Dipole and we identify it with this made.
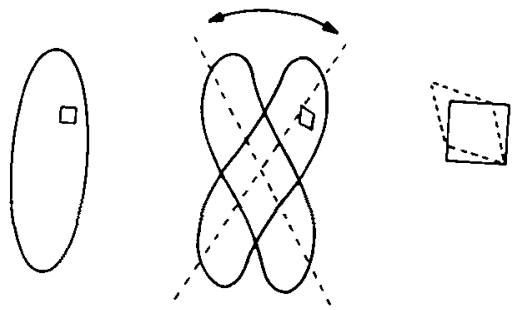

Fig. 4 Pictorial impression of Giant Angle Dipole motion

However, the correspondence to the collective model picture should really be sought in the subset of fragmented states having similar underlying structure. Of the states carrying any sizable B(M1) strength, only those at energies $2.67,3.04,3.65$ and 4.89 Mev seem to have physical structure related to the dominant component situated at 3.41 Mev. Common to all of these states is their small spin flip to orbital M1 strength, and the fact that their overlaps noticeably increase if shear admixture is allowed. The differences appear in the amount of isovector and isoscaler admixture present in each state. Although none of these states have overlaps (7) as 1 arge as that for the dominant component, typically they lie in the range $(36 \%-48 \%)$. The strengths shown in fig.l should however be thought of as a guide only, since basis truncation effects may still produce some changes $14,5 /$.

\section{ACCESSIBLE INFORMATION}

The experimental confirmation of this state allows us access to a wealth of information about the nucleus. Knowledge of new inertial parameters and restoring forces related to both rotation and deformation are required for the description of the Giant Angle Dipale and as already demonstrated /3/, the latter may differ considerably from that obtained from a pure contra rotation picture. The character of the mode, as a sum of contra rotation and shear, accords not only with that expected from an incompressible fluid/16/. The shear isospin admixture may also be echoed in the observations that neutron and proton transfer times are different $/ 17 /$, indicating response differences between neutrons and protons to deformation changes, and hence collective flow properties. The form of tie fragmentation spectrum also promises to shed light on the way the nucleus responds to shear. A picture of breakup, at least at low energy, may be possible to read off. Recent measurements/13/ imply very small spin-flip contributions to this state. Since the spin-orbit force plays a crucial role here, in these measurements we may have an instrument giving us a precise handle on its strength. In fact more generally the Giant Angle Dipole already appears to act as a sort of test bench with which one will be able to "weed out" some types of effective interactions.

A rich source for future nuclear structure physics is clearly in prospect.

\section{REFERENCES}

1) "A possible vibrational mode in heavy nuclei", talk presented by R.R.Hilton at International Conference on Nuclear Structure", Dubna, June 1976 . 
"Description of a possible Giant Angle Dipole excitation in deformed nuclei", talk presented by R.R.Hilton at Les Houches Summer School in Theoretical Physics,July-Aug. 1977

"The Giant Angle Dipale", talk presented by R.R.Hilton at DPG meeting, Heidelberg, March 1978 Proc. p823.

"New collective excitations in deformed nuclei", talk presented by R.R.Hilton at joint DPG-OPG meeting, Munich 1980 Proc. p1218.

"Collective aspects of the Shell Model", contributed paper by R.R.Hilton to the International Conference on Nuclear Physics, Berkley, August 1980 Proc. p276.

2) R.R.Hilton, Z. Physik A316 (1984) 471

3) R.R.Hilton J. de Phys. (Paris) Co Supplement to Na.6 45 June (1984) P255

4) R.R.Hilton, M.Faber, S.Iwasaki, H.J.Mang and P.Ring: Proc. "Phase Space Approach to Nuclear Dynamics" Trieste, Sept. 1985 p749, Pub. World Scientific 1986, Eds. M.di toro, W.Noerenberg, M.Rosina, S.Stringari

5) R.R.Hilton, M.Faber, 5.Iwasaki, H.J.Mang and P.Ring: Proceedings "Microscopic Approach to Nuclear Structure Calculations" , Sorrento, May 1986. Editrice Compositori-Bologna, p357, 1986 Ed. A.Covello

6) N. Lo Iudice and F. Palumbo, Phys. Rev. Letts. 41 (1978) 1532 and Nuc1. Phys. A326 (1979) 193

7) T.Suzuki and D.J. Rowe, Nucl. Phys. A289 (1977) 461

8) D. Bohle et al, Phys. Letts. 1378 (1984) 27 and 1488 (1984) 260

9) A.Richter, "Nuclear Structure 1985", Proc. of Niels Bohr Centennial Conference, Copenhagen May, (1985) P469 Eds. R. Broglia, G.Hagemann, B. Herskind. North Holl and 1985

10) U.E.P.Berg et al Phys Letts. 149B (1984) 59 and J. de Phys. (Paris) C4 No.3, 45, March 1984, p359 and Proc. DPG meeting, Munich, March 1985 p536

11) C. Djalali et al Phys. Letts. 1648 (1985) 269

12) J.A.Carr, F.Petrovich, M.J.Threapleton, O.Scholten and H. McManus Phys. Rev. Letts. 54 (1985) 881

13) C. Wesselborg et al Z. Phys. A 323 (1986) 485

14) D.M.Brink, Nucl. Phys. 4 (1957) 215

15) P.Ring and P.Schuck, "The Nuclear Many Body Problem", Springer Verlag 1980

16) G.Stokes Camb. Phil. Trans. Viii (1845) Papers i, 80.

17) H.J.Körner, Proc of Symposium "10 Years of Uranium Beam at the UNILAC", GSI Darmstadt, April 1986, p158. Pub GSI Nov. 1986 ISSN:0171-4546 Eds. N.Angert, P.Kienle 